\title{
Berezin-Toeplitz quantization and the least unsharpness principle
}

\author{
Louis $\operatorname{Ioos}^{1}$, David Kazhdan and Leonid Polterovich
}

April 23, 2020

\begin{abstract}
We show that compatible almost-complex structures on symplectic manifolds correspond to optimal quantizations.
\end{abstract}

\section{Contents}

1 Introduction 2

2 Berezin-Toeplitz quantization 4

3 Unsharpness cocycle 8

4 The least unsharpness principle 11

5 Proof of the main Theorem 16

6 Case study: $S U(2)$ - equivariant quantizations 22

7 The unsharpness cocycle is of order $(1,1)$

8 Discussion and questions 27

8.1 Historical remarks on unsharpness . . . . . . . . . . 27

8.2 Least unsharpness surfaces and pseudo-holomorphic curves . . 28

8.3 On classification of Berezin-Toeplitz quantizations . . . . . . . 29

\footnotetext{
${ }^{1}$ Partially supported by the European Research Council Starting grant 757585
} 


\section{Introduction}

A Riemannian metric on a symplectic manifold $(M, \omega)$ is $\omega$-compatible if it can be written as

$$
g_{\omega, J}(\cdot, \cdot):=\omega(\cdot, J \cdot),
$$

where $J$ is an almost complex structure on $M$. Vice versa, an almost complex structure $J$ is $\omega$-compatible if the bilinear form (1) is a Riemannian metric. Compatible geometric structures were introduced as an effective auxiliary tool for detecting rigidity phenomena on symplectic manifolds [19]. In the present paper we show that these structures naturally arise from the perspective of mathematical physics. Loosely speaking, they correspond to "optimal" quantizations, the ones minimizing a natural physical quantity called unsharpness, which is one of the main characters of this paper (see Section 3 below).

Quantization is a mathematical recipe behind the quantum-classical correspondence, a fundamental physical principle stating that quantum mechanics contains classical mechanics in the limiting regime when the Planck constant $\hbar$ tends to zero [17]. There exist two different, albeit related mathematical models of this principle. Assume that the classical phase space is represented by a closed (i.e., compact without boundary) symplectic manifold $(M, \omega)$. The first model, deformation quantization, is a formal associative deformation

$$
f * g=f g+\hbar c_{1}(f, g)+\hbar^{2} c_{2}(f, g)+\cdots
$$

of the multiplication on the space $C^{\infty}(M)$ of smooth real functions on $M$ such that $f * g-g * f=i \hbar\{f, g\}+\mathcal{O}\left(\hbar^{2}\right)$, where $\{\cdot, \cdot\}$ stands for the Poisson bracket [4]. The operation $*$ is called the star-product and the Planck constant $\hbar$ plays the role of a formal deformation parameter.

The second model, geometric quantization, is described as a linear correspondence $f \mapsto T_{\hbar}(f)$ between classical observables, i.e., real functions $f$ on the phase space $M$, and quantum observables, i.e., Hermitian operators on a complex Hilbert space. This correspondence is assumed to respect, in the leading order as $\hbar \rightarrow 0$, a number of basic operations. In the present paper, we focus on Berezin-Toeplitz quantizations [5, 9, 20, 10, 29, 35, 14, whose distinctive feature is to send non-negative functions to non-negative operators (see Section 2). The known models of Berezin-Toeplitz quantization on closed symplectic manifolds (see the discussion following Theorem 2.7) determine a deformation quantization [9, 34, 20], and are provided by 
certain auxiliary data involving in particular an almost complex structure $J$ compatible with the symplectic form on the phase space. While deformation quantizations of closed symplectic manifolds are completely classified up to a natural equivalence, the classification of Berezin-Toeplitz quantizations is not yet completely understood (see however [27] for the relation between the two).

The main finding of the present paper is that conversely, any BerezinToeplitz quantization, defined through natural axioms presented in Section 2. gives rise in a canonical way to a Riemannian metric on the phase space. Specifically, we make the natural assumption that there exists of a complexvalued bi-differential operator $c: C^{\infty}(M) \times C^{\infty}(M) \rightarrow C^{\infty}(M, \mathbb{C})$ such that the $\mathbb{C}$-linear extension of $T_{\hbar}$ satisfies

$$
T_{\hbar}(f) T_{\hbar}(g)=T_{\hbar}(f g)+\hbar T_{\hbar}(c(f, g))+\mathcal{O}\left(\hbar^{2}\right),
$$

for all $f, g \in C^{\infty}(M)$ as $\hbar \rightarrow 0$, and we show that this induces a Riemannian metric $G$ on $M$ by the formula

$$
c(f, g)=-\frac{1}{2} G(\operatorname{sgrad} f, \operatorname{sgrad} g),
$$

where sgrad $f$ stands for the Hamiltonian vector field of a function $f$ on $M$. (see Theorem 4.1(I) below).

Leaving precise definitions for Section 4, let us discuss the above-mentioned results informally and present a motivation coming from physics. To this end recall that it is classically known, starting from the Groenewold-van Hove theorem, that a Berezin-Toeplitz correspondence cannot be a genuine morphism between the Lie algebras of functions and operators. We focus on yet another constraint on the precision of Berezin-Toeplitz quantizations, which we call unsharpness, and which is governed by the Riemannian metric $G$ defined above. The notion of unsharpness is closely related to the Heisenberg uncertainty principle. It comes from an analogy between quantization and measurement based on the formalism of positive operator valued measures (POVMs), which serves both subjects, and which we briefly recall in Section 2. The unsharpness metric is a particular instance of the noise operator [12] describing, loosely speaking, the increment of variances in the process of quantization (see the discussion p.13).

In this language, we propose the least unsharpness principle, a variational principle selecting quantizations whose unsharpness metric has minimal possible volume on phase space. It turns out that the least unsharpness volume 
equals the symplectic volume (see Theorem 4.1(II) below), so that a quantization satisfying the least unsharpness principle determines a compatible almost complex structure $J$ on $(M, \omega)$, in the sense that its unsharpness metric satisfies $G=g_{\omega, J}$ as in (11). We refer to Section 3 for basic properties of unsharpness, while existence of the unsharpness metric and the least unsharpness principle are stated in Section 4 and proved in Section 5 .

The unsharpness metric is a natural geometric invariant of a BerezinToeplitz quantization, and can be seen as a first step towards classification. As a case study, we show in Section [6 that for $S U(2)$-equivariant quantizations of the two-dimensional sphere, the unsharpness metric completely determines the quantization up to conjugation and up to second order as $\hbar \rightarrow 0$. Further comments on classification can be found in Section 8.3.

Some historical remarks are in order. A canonical appearance of Riemannian geometry in quantization was discussed on a number of occasions in physical literature. Anandan and Aharonov [1] and Ashtekar and Schilling [3] developed a geometric approach to quantum mechanics based on the FubiniStudy metric on the projective space of pure quantum states. Klauder (see, e.g., [28]) studied a model of a path-integral quantization where the role of a metric was to define a Brownian motion on the phase space. The idea of selecting optimal quantizations as those possessing the least uncertainty goes back to Gerhenstaber [18. He deals with quantizations which do not necessarily preserve positivity, and his least uncertainty principle implies that unsharpness identically vanishes on some restricted class of observables (see Section 8.1 for further discussion). Finally, while classification of equivariant quantizations is known in the context of deformation quantization [2, 7], our setting, including the notion of equivalence, is substantially different. The case of $S U(2)$-equivariant Berezin-Toeplitz quantizations of the sphere which we settle in Section [6 is, to the best of our knowledge, the first one where a complete classification is currently available.

\section{Berezin-Toeplitz quantization}

Given a finite-dimensional complex Hilbert space $H$, we write $\mathcal{L}(H)$ for the space of all Hermitian operators (representing quantum observables), and $\mathcal{S}(H) \subset \mathcal{L}(H)$ for the subset of all non-negative trace-one Hermitian operators (representing quantum states). 
Definition 2.1. An $\mathcal{L}(H)$-valued positive operator valued measure (POVM) on a set $M$ equipped with a $\sigma$-algebra $\mathcal{C}$ is a map $\Gamma: \mathcal{C} \rightarrow \mathcal{L}(H)$ which satisfies the following conditions:

- $\Gamma(\emptyset)=0$ and $\Gamma(M)=\mathbb{1}$;

- $\Gamma(X) \geq 0$ for every $X \in \mathcal{C}$;

- $\left(\sigma\right.$-additivity) $\Gamma\left(\bigsqcup_{i \in \mathbb{N}} X_{i}\right)=\sum_{i \in \mathbb{N}} \Gamma\left(X_{i}\right)$ for any sequence of pair-wise disjoint subsets $\left\{X_{i} \in \mathcal{C}\right\}_{i \in \mathbb{N}}$.

According to [16], for every $\mathcal{L}(H)$-valued POVM measure $\Gamma$, there exist a probability measure $\alpha$ on $(M, \mathcal{C})$ and a measurable function $F: M \rightarrow \mathcal{S}(H)$ such that

$$
d \Gamma=n F d \alpha,
$$

where $n=\operatorname{dim}_{\mathbb{C}} H$.

Remark 2.2. In the context of quantization, the state $F_{x} \in \mathcal{S}(H)$ is called the coherent state associated with $x \in M$, and describes the quantization of a classical particle sitting at $x \in M$ in phase space.

For any real classical observable $f \in L^{1}(M, \alpha)$, we define the quantization $T(f)$ as the integral

$$
T(f):=\int_{M} f d \Gamma \in \mathcal{L}(H) .
$$

The dual map $T^{*}: \mathcal{L}(H) \rightarrow L^{\infty}(M)$ with respect to the scalar product $((A, B)):=\operatorname{tr}(A B), A, B \in \mathcal{L}(H)$ satisfies $T^{*}(A)(x)=n \operatorname{tr}(A F(x))$, for any $x \in M$ and $A \in \mathcal{L}(H)$.

Remark 2.3. For a quantum observable $A$, the function $T^{*}(A) \in L^{\infty}(M)$ has a natural interpretation as the classical observable whose value at $x \in M$ is the expectation value of $A$ at the associated coherent state $F_{x}$. Thus, we call $T^{*}(A)$ the dequantization of the quantum observable $A \in \mathcal{L}(H)$.

Definition 2.4. The composition

$$
\begin{aligned}
\mathcal{B}:=\frac{1}{n} T^{*} T: L^{1}(M, \alpha) & \longrightarrow L^{\infty}(M), \\
f(x) & \longmapsto \operatorname{tr}\left(T(f) F_{x}\right)
\end{aligned}
$$

is called the Berezin transform associated to the POVM $\Gamma$. 
Remark 2.5. The Berezin transform can be interpreted as quantization followed by dequantization. It is a measure of the blurring induced by quantization.

To study the quantum-classical correspondence, we need to introduce a parameter $\hbar$ in the above story, which can be thought as the Planck constant, and from which we recover the laws of classical mechanics as $\hbar \rightarrow 0$. This is given a precise meaning via the following definition.

Definition 2.6. Let $(M, \omega)$ be a closed connected symplectic manifold of dimension $2 d$ and $\mathcal{C}$ be the $\sigma$-algebra of its Borel sets in $M$. A BerezinToeplitz quantization of $M$ is the following data:

- a subset $\Lambda \subset \mathbb{R}_{>0}$ having 0 as limit point ;

- a finite-dimensional complex Hilbert space $H_{\hbar}$ for each $\hbar \in \Lambda$;

- an $\mathcal{L}\left(H_{\hbar}\right)$-valued positive operator valued measures $\Gamma_{\hbar}$ on $M$ for each $\hbar \in \Lambda$,

such that the Toeplitz map $T_{\hbar}: C^{\infty}(M, \mathbb{C}) \rightarrow \operatorname{End}\left(H_{\hbar}\right)$ induced for all $\hbar \in \Lambda$ by the quantization map (4) is surjective and satisfies the following estimates, uniformly in the $C^{N}$-norms of $f, g \in C^{\infty}(M)$ for some $N \in \mathbb{N}$ :

(P1) (norm correspondence)

$$
\|f\|-\mathcal{O}(\hbar) \leq\left\|T_{\hbar}(f)\right\|_{\mathrm{op}} \leq\|f\|,
$$

where $\|\cdot\|_{o p}$ is the operator norm and $\|f\|:=\max _{x \in M}|f(x)|$;

(P2) (bracket correspondence)

$$
\left\|-\frac{i}{\hbar}\left[T_{\hbar}(f), T_{\hbar}(g)\right]-T_{\hbar}(\{f, g\})\right\|_{\mathrm{op}}=\mathcal{O}(\hbar),
$$

where $[\cdot, \cdot]$ stands for the commutator and $\{\cdot, \cdot\}$ for the Poisson bracket 1 ;

(P3) (quasi-multiplicativity) There exists a bi-differential operator $c: C^{\infty}(M) \times C^{\infty}(M) \rightarrow C^{\infty}(M, \mathbb{C})$ such that

$$
\left\|T_{\hbar}(f) T_{\hbar}(g)-T_{\hbar}(f g)-\hbar T_{\hbar}(c(f, g))\right\|_{o p}=\mathcal{O}\left(\hbar^{2}\right) .
$$

\footnotetext{
${ }^{1}$ Our convention for the Poisson bracket is $\{f, g\}:=-\omega(\operatorname{sgrad} f, \operatorname{sgrad} g)$ for all $f, g \in$ $C^{\infty}(M)$, where $\operatorname{sgrad} f$ is the Hamiltonian vector field of $f$ defined by $\iota_{\operatorname{sgrad} f} \omega+d f=0$.
} 
(P4) (trace correspondence)

$$
\operatorname{tr}\left(T_{\hbar}(f)\right)=(2 \pi \hbar)^{-d} \int_{M} f R_{\hbar} d \mu,
$$

where $R_{\hbar} \in C^{\infty}(M)$ satisfies $R_{\hbar}=1+\mathcal{O}(\hbar)$, and $d \mu=\frac{\omega^{d}}{d !}$ is the symplectic volume on $M$;

(P5) (reversibility) The maps $\mathcal{B}_{\hbar}: C^{\infty}(M) \rightarrow C^{\infty}(M)$ induced by the Berezin transform (5) satisfy

$$
\mathcal{B}_{\hbar}(f)=f+\mathcal{O}(\hbar) .
$$

By uniformly in the $C^{N}$-norms of $f, g \in C^{\infty}(M)$, we mean that there exists a constant $C>0$ such that, in axioms (P2) and (P3) and for $k=1,2$ respectively, the remainders satisfy

$$
\left|\mathcal{O}\left(\hbar^{k}\right)\right| \leq C \hbar^{k}\|f\|_{C^{N}}\|g\|_{C^{N}},
$$

while in axioms (P1) and (P5), the remainders satisfy $|\mathcal{O}(\hbar)| \leq C \hbar\|f\|_{C^{N}}$. Writing the density (3) associated to $\Gamma_{\hbar}$ in the form

$$
d \Gamma_{\hbar}(x)=n_{\hbar} F_{\hbar, x} d \alpha_{\hbar}(x),
$$

the trace correspondence (P4) implies

$$
n_{\hbar}=\frac{\operatorname{Vol}(M, \omega)+\mathcal{O}(\hbar)}{(2 \pi \hbar)^{d}},
$$

and

$$
d \alpha_{\hbar}=\frac{1+\mathcal{O}(\hbar)}{\operatorname{Vol}(M, \omega)} d \mu .
$$

where $\operatorname{Vol}(M, \omega)>0$ denotes the symplectic volume of $(M, \omega)$.

The existence of a Berezin-Toeplitz quantization is a highly non-trivial result. To discuss it, recall that an almost complex structure $J$ on $M$ is $\omega$-compatible if the form $G_{J}:=\omega(\cdot, J \cdot)$ is a Riemannian metric on $M$. We refer to $\left(M, \omega, J, G_{J}\right)$ as an almost-Kähler structure on $M$.

Theorem 2.7. If the closed symplectic manifold $(M, \omega)$ is quantizable, i.e., if the cohomology class $[\omega] /(2 \pi)$ is integral, then every $\omega$-compatible almostcomplex structure $J$ defines a Berezin-Toeplitz quantization of $(M, \omega)$, with $\Lambda=\{1 / k\}_{k \in \mathbb{N}}$. 
In the case of Kähler manifolds, i.e., if we assume additionally that the almost complex structure $J$ is integrable, there is a canonical construction of a Berezin-Toeplitz quantization, where the Hilbert spaces $H_{\hbar}$ consist of the global holomorphic sections of a holomorphic Hermitian line bundle with Chern curvature equal to $-2 \pi i k \omega$, with $\hbar=1 / k$, and the associated Toeplitz map $T_{\hbar}$ sends $f \in C^{\infty}(M)$ to the multiplication by $f$ followed by the orthogonal $L_{2}$-projection on holomorphic sections. In this context, Theorem 2.7 has been established by Bordemann, Meinrenken and Schlichenmaier in [9], using the theory of Toeplitz structures developed by Boutet de Monvel and Guillemin in [11]. The fact that this theory extends to the almost-Kähler case was proved in a series of papers by Guillemin [20], Borthwick and Uribe [10], Schiffman and Zelditch [36, Ma and Marinescu [29], Charles [14] and the first named author, Lu, Ma and Marinescu [23]. The dependence of the remainders in terms of the derivatives of the functions is discussed in [15].

\section{Unsharpness cocycle}

In this section, we study general properties of the bi-differential operator $c: C^{\infty}(M) \times C^{\infty}(M) \rightarrow C^{\infty}(M)$ from the quasi-multiplicativity property (P3) of a Berezin-Toeplitz quantization. First note that norm correspondence (P1) implies that, if an asymptotic expansion such as the one appearing in (P3) holds, then it is unique, and in particular, the bi-differential operator $c$ is uniquely defined. Then the associativity of the composition of operators implies that $c$ is a Hochschild cocycle, meaning that for all $f_{1}, f_{2}, f_{3} \in C^{\infty}(M)$, we have

$$
f_{1} c\left(f_{2}, f_{3}\right)-c\left(f_{1} f_{2}, f_{3}\right)+c\left(f_{1}, f_{2} f_{3}\right)-c\left(f_{1}, f_{2}\right) f_{3}=0 .
$$

Denote by $c_{-}$and $c_{+}$its anti-symmetric and symmetric parts, respectively:

$$
c_{-}(f, g):=\frac{c(f, g)-c(g, f)}{2} \text { and } \quad c_{+}(f, g):=\frac{c(f, g)+c(g, f)}{2} .
$$

By the bracket correspondence (P2), we have $T_{\hbar}\left(2 c_{-}(f, g)-i\{f, g\}\right)=\mathcal{O}(\hbar)$, and hence by the norm correspondence (P1), we get the formula

$$
c_{-}(f, g)=\frac{i}{2}\{f, g\} \text {. }
$$

Thus the anti-symmetric part $c_{-}$(responsible for the non-commutativity of quantum observables) does not depend on a choice of a quantization. In 
contrast, the symmetric part $c_{+}$does depend on a choice of a quantization. By the quasi-multiplicativity property (P3), the cocycle $c_{+}$associated to a Berezin-Toeplitz quantization measures its failure of being a multiplicative morphism on Poisson-commutative subspaces of $C^{\infty}(M)$. From formula (10) and basic properties of the Poisson bracket, we know that $c^{-}$, hence also $c^{+}$, satisfy formula (9) for a Hochschild cocycle.

Definition 3.1. We say that $c_{+}$is the unsharpness cocycle of a quantization or simply its unsharpness.

Note that by formula (41), the operator $T_{\hbar}(f) \in \operatorname{End}\left(H_{\hbar}\right)$ is Hermitian if and only if $f \in C^{\infty}(M, \mathbb{C})$ is real valued, and as the square of a Hermitian operator is Hermitian, the quasi-multiplicativity property (P3) then shows that $c^{+}: C^{\infty}(M) \times C^{\infty}(M) \rightarrow C^{\infty}(M)$ is a real-valued bi-differential operator. It is also a symmetric Hochschild cocycle, so that as explained e.g. in [32, Prop. 2.14], it is a differential Hochschild coboundary. This means that there exists a real-valued differential operator $a: C^{\infty}(M) \rightarrow C^{\infty}(M)$ such that

$$
c_{+}(f, g)=a(f g)-f a(g)-g a(f) .
$$

for all $f, g \in C^{\infty}(M)$. Since $T_{\hbar}(1)=1$, we have that $c_{+}(1,1)=0$, and therefore $a(1)=0$. Note that $a$ is determined up to its first order part. The following result shows that the positivity preserving property imposes a strong condition on $c_{+}$.

Theorem 3.2. The bi-differential operator $c_{+}$is of order $(1,1)$.

The proof is given in Section 7 below. Theorem 3.2 sheds light on the differential operator $a$ appearing in the coboundary formula (111). In fact, let us choose some Darboux coordinates $U \subset X$, and take $f, g \in C^{\infty}(M)$ with compact support in $U$. In these coordinates, we can write

$$
c_{+}(f, g)=\sum_{j, k=1}^{2 d} a_{j k} \partial_{j} f \partial_{k} g,
$$

with smooth $a_{j k}=a_{k j} \in C^{\infty}(U)$ for each $1 \leq j, k \leq 2 d$. Then one can choose the differential operator

$$
a:=\frac{1}{2} \sum_{j, k=1}^{2 d} \partial_{j}\left(a_{j k} \partial_{k}\right) .
$$


in the coboundary formula (11). Using integration by parts, we see that (13) is symmetric with respect to the canonical $L^{2}$-scalar product on $C^{\infty}(M)$ associated to the symplectic volume, and as the differential operator $a$ is determined up to its first order part, it is the unique such choice.

Example 3.3. Assume that $(M, \omega)$ is quantizable and equipped with an almost-Kähler structure $\left(M, \omega, J, G_{J}\right)$. Then the induced Berezin-Toeplitz quantization of Theorem 2.7 satisfies

$$
c_{+}(f, g)=-\frac{1}{2}(\nabla f, \nabla g)
$$

where the gradient and the product are defined with respect to $G_{J}$. Using that

$$
\Delta(f g)+2(\nabla f, \nabla g)=f \Delta g+g \Delta f,
$$

where $\Delta$ is the (positive) Laplace-Beltrami operator associated with $G_{J}$, the differential operator in the coboundary formula (11) can then be chosen to be $a=\Delta / 4$, and by basic properties of $\Delta$, it is the unique $L^{2}$-symmetric choice with respect to the symplectic volume form, as it coincides with the Riemannian volume form of $G_{J}$. Formula (14) can be found in [39, p. 257] for the Kähler case and in [22, 23] for the almost-Kähler case. Using the $J$-invariance of the metric and the relation $J \operatorname{sgrad} f=-\nabla f$ between Hamiltonian vector field and gradient of a function $f \in C^{\infty}(M)$ for an $\omega$-compatible metric, formula (14) translates into

$$
c_{+}(f, g)=-\frac{1}{2} G_{J}(\operatorname{sgrad} f, \operatorname{sgrad} g) .
$$

Example 3.4. We now give an example of a Berezin-Toeplitz quantization whose unsharpness cocycle $c_{+}$is not of the form (16) for some almost-Kähler structure on $(M, \omega)$. This example serves as a paradigm for the construction presented in the proof of one of our main results, Theorem 4.1(III) below. Assume $(M, \omega)$ quantizable and equipped with an almost-Kähler structure $\left(M, \omega, J, G_{J}\right)$, and consider the induced Berezin-Toeplitz quantization of Theorem 2.7. Fix $t>0$, and using the notations of Example 3.4, consider for any $\hbar \in \Lambda=\{1 / k\}_{k \in \mathbb{N}}$ the map $T_{\hbar}^{(t)}: C^{\infty}(M) \rightarrow \mathcal{L}\left(H_{\hbar}\right)$ defined for any $f \in C^{\infty}(M)$ by

$$
T_{\hbar}^{(t)}(f):=T_{\hbar}\left(e^{-t \hbar \Delta} f\right) .
$$

Observe that the heat flow preserves positivity, so that $T_{\hbar}^{(t)}$ is in fact the quantization map (4) induced by a POVM construction. Then from the 
classical small time asymptotic expansion of the heat kernel (see e.g. [6, Th. 2.29, (2.8)]), as $\hbar \rightarrow 0$, we have

$$
e^{-t \hbar \Delta} f=f-t \hbar \Delta f+\mathcal{O}\left(\hbar^{2}\right)\|f\|_{C^{4}}
$$

and this implies in particular that all the axioms of Definition 2.6 hold. Let us now calculate the associated unsharpness cocycle, denoted by $c_{+}^{(t)}$. For any $\hbar \in \Lambda$ and $A, B \in \operatorname{End}\left(H_{\hbar}\right)$, put $A \bullet B:=\frac{1}{2}(A B+B A)$, and recall formula (15) for the Laplace-Beltrami operator. Then as $\hbar \rightarrow 0$, we have

$$
\begin{aligned}
& T_{\hbar}^{(t)}(f) \bullet T_{\hbar}^{(t)}(g)=T_{\hbar}(f) \bullet T_{\hbar}(g)-t \hbar T_{\hbar}(f \Delta g+g \Delta f)+\mathcal{O}\left(\hbar^{2}\right) \\
& =T_{\hbar}(f g)-\hbar T_{\hbar}\left(\frac{1}{2}(\nabla f, \nabla g)+t(\Delta(f g)+2(\nabla f, \nabla g))\right)+\mathcal{O}\left(\hbar^{2}\right) \\
& =T_{\hbar}(f g)+\hbar T_{\hbar}^{(t)}\left(-\left(\frac{1}{2}+2 t\right)(\nabla f, \nabla g)\right)+\mathcal{O}\left(\hbar^{2}\right)
\end{aligned}
$$

so that, recalling that the quasi-multiplicativity property (P3) determines the unsharpness cocycle uniquely via norm correspondence (P1), we get

$$
\begin{aligned}
c_{+}^{(t)}(f, g) & =-\left(\frac{1}{2}+2 t\right)(\nabla f, \nabla g) \\
& =-\frac{1}{2}(1+4 t) G_{J}(\operatorname{sgrad} f, \operatorname{sgrad} g) .
\end{aligned}
$$

In particular, we see that $c_{+}^{(t)}$ is of the form (16) for the Riemannian metric $G^{(t)}:=(1+4 t) G_{J}$ on $M$, whose volume is strictly bigger than the volume of the almost-Kähler metric $G_{J}$. As the volume of an almost-Kähler metric is always equal to the symplectic volume of $(M, \omega)$, we see from (3.3) that $c_{+}^{(t)}$ is not the unsharpness cocycle of a Berezin-Toeplitz quantization coming from Theorem 2.7.

\section{The least unsharpness principle}

In this section, we state the main theorem on unsharpness of BerezinToeplitz quantizations, which we call the least unsharpness principle, and discuss its physical meaning. 
Recall from Theorem 3.2 that the unsharpness cocycle $c_{+}$of a BerezinToeplitz quantization is a bi-differential operator of order $(1,1)$, so that there exists a bilinear symmetric form $G$ on $T M$ such that

$$
c_{+}(f, g)=:-\frac{1}{2} G(\operatorname{sgrad} f, \operatorname{sgrad} g),
$$

where sgrad $f, \operatorname{sgrad} g$ denote the Hamiltonian vector fields of $f, g \in C^{\infty}(M, \mathbb{R})$. Our main result provides a description of this bilinear form $G$.

Theorem 4.1. Let $(M, \omega)$ be a closed symplectic manifold.

(I) For every Berezin-Toeplitz quantization of $M$, the form $G$ is a Riemannian metric on $M$ which can be written as the sum

$$
G=\omega(\cdot, J \cdot)+\rho(\cdot, \cdot),
$$

where $J \in \operatorname{End}(T M)$ is a compatible almost complex structure on $(M, \omega)$ and $\rho$ is a non-negative symmetric bilinear form on $T M$.

(II) We have $\operatorname{Vol}(M, G) \geq \operatorname{Vol}(M, \omega)$, with equality if and only if $\rho \equiv 0$.

(III) If $(M, \omega)$ is quantizable, then every Riemannian metric of the form (21) arises from some Berezin-Toeplitz quantization.

The proof is given in Section 5. Let us mention that the proof of item (III) of the theorem is modeled on Example 3.4 above and is constructive. We produce the desired Berezin-Toeplitz quantization with the unsharpness metric given by (21) as the composition of the almost-Kähler quantization associated to $(\omega, J)$ and an explicit, albeit non-canonical, Markov operator depending on all the data including $\rho$.

Remark 4.2. For a given metric $G$ on $M$, the decomposition (21) is in general not unique. However, as the proof of Theorem4.1(I) will show, there exists a unique $\omega$-compatible almost complex structure $J$ which additionally is $G$-orthogonal, i.e., $G(J \xi, J \eta)=G(\xi, \eta)$ for all $\xi, \eta \in T M$. Furthermore, for such a $G$, the symmetric bilinear form $\rho(\xi, \eta)=G(\xi, \eta)-\omega(\xi, J \eta)$ is non-negative, thus providing decomposition (21). 
Before going to the proof of Theorem 4.1 in the next Section, let us first discuss the physical meaning of the unsharpness cocyle $c^{+}$associated with a Berezin-Toeplitz operator, which shows from general principles that it is at least non-negative. With every quantum state $\theta \in \mathcal{S}\left(H_{\hbar}\right)$ one associates a classical state (called the Husimi measure), which is the probability measure $\mu_{\theta}$ on $M$ such that

$$
\int_{M} f d \mu_{\theta}=\operatorname{tr}\left(T_{\hbar}(f) \theta\right), f \in C^{\infty}(M)
$$

This equality can be interpreted as follows: the expectation of any classical observable $f$ in the classical state $\mu_{\theta}$ coincides with the expectation of the corresponding quantum observable $T_{\hbar}(f)$ in the state $\theta$. What happens with variances? It turns out that the quantum variance is in general bigger than the classical one. More precisely, we have that

$$
\begin{aligned}
\operatorname{Var}\left(f, \mu_{\theta}\right) & =\int_{M} f^{2} d \mu_{\theta}-\left(\int f d \mu_{\theta}\right)^{2}, \\
\mathbb{V a r}\left(T_{\hbar}(f), \theta\right) & =\operatorname{tr}\left(T_{\hbar}(f)^{2} \theta\right)-\left(\operatorname{tr}\left(T_{\hbar}(f) \theta\right)\right)^{2},
\end{aligned}
$$

and hence

$$
\operatorname{Var}\left(f, \mu_{\theta}\right)=\operatorname{Var}\left(T_{\hbar}(f), \theta\right)+\operatorname{tr}\left(\Delta_{\hbar}(f) \theta\right),
$$

where

$$
\Delta_{\hbar}(f):=T_{\hbar}\left(f^{2}\right)-T_{\hbar}(f)^{2} .
$$

The operator $\Delta_{\hbar}(f)$ is called the noise operator (see e.g. [12]), whose main property is that it is a non-negative operator. It describes the increase of variances, which can be interpreted as the unsharpness of the quantization. Then by the quasi-multiplicativity property (P3), we have

$$
\Delta_{\hbar}(f)=-\hbar T_{\hbar}\left(c_{+}(f, f)\right)+\mathcal{O}\left(\hbar^{2}\right) .
$$

Look at the expectation of $\Delta_{\hbar}(f)$ at the coherent state $F_{\hbar, x}$ of Remark 2.2 associated to $\Gamma_{\hbar}$,

$$
\begin{aligned}
\operatorname{tr} & \left(\Delta_{\hbar}(f) F_{\hbar, x}\right)=-\hbar \operatorname{tr}\left(T_{\hbar}\left(c_{+}(f, f)\right) F_{\hbar, x}\right)+\mathcal{O}\left(\hbar^{2}\right) \\
& =-\hbar \mathcal{B}_{\hbar}\left(c_{+}(f, f)\right)(x)+\mathcal{O}\left(\hbar^{2}\right) \\
& =-\hbar c_{+}(f, f)(x)+\mathcal{O}\left(\hbar^{2}\right) .
\end{aligned}
$$


This explains the name of unsharpness cocycle for $c^{+}$. Since the noise operator is non-negative, we get the following fundamental property of the unsharpness cocycle,

$$
-c_{+}(f, f)(x) \geq 0 \text { for all } x \in M .
$$

This shows that the symmetric bilinear form $G$ defined in equation (20) is at least semi-positive. This property is the first step towards the proof of Theorems 4.1, showing that $G$ is in fact a Riemannian metric, called the unsharpness metric of the quantization. Note that this property is also at the basis of the proof of Theorem 3.2 .

Define the total unsharpness of a Berezin-Toeplitz quantization as the volume of the phase space $M$ with respect to the unsharpness metric. With this language, statement (II) of Theorem 4.1 can be interpreted as the least unsharpness principle: the minimal possible total unsharpness equals the symplectic volume, and the least unsharpness metrics are induced by compatible almost-complex structures on $M$.

Remark 4.3. Let us assume that the Berezin transform admits an asymptotic expansion up to the first order as $\hbar \rightarrow 0$ of the following form for all $f \in C^{\infty}(M)$,

$$
\mathcal{B}_{\hbar}(f)=f+\hbar D f+\mathcal{O}\left(\hbar^{2}\right)
$$

where $D$ is a differential operator, stenghtening the reversibility property (P5). Then by Definition 2.4 of the Berezin transform, formula (4) for the quantization map and the expansion (8) for $\alpha_{\hbar}$, for all $f, g \in C^{\infty}(M)$, we have

$$
\begin{aligned}
\frac{1}{n_{\hbar}} \operatorname{tr}\left(T_{\hbar}(f) T_{\hbar}(g)\right) & =\int_{M} \mathcal{B}_{\hbar}(f) g d \alpha_{\hbar} \\
& =\int_{M} f g d \alpha_{\hbar}+\hbar \int_{M}(D f) g d \mu+\mathcal{O}\left(\hbar^{2}\right) .
\end{aligned}
$$

On the other hand, by the quasi-mutliplicativity property (P3), using formula (10) and basic properties of the Poisson bracket, we get

$$
\begin{aligned}
& \frac{1}{n_{\hbar}} \operatorname{tr}\left(T_{\hbar}(f) T_{\hbar}(g)\right)=\int_{M}\left(f g+\hbar c(f, g)+\mathcal{O}\left(\hbar^{2}\right)\right) d \alpha_{\hbar} \\
& =\int_{M} f g d \alpha_{\hbar}+\hbar \int_{M} c_{+}(f, g) d \mu+\mathcal{O}\left(\hbar^{2}\right) .
\end{aligned}
$$


Then taking $f, g \in C^{\infty}(M)$ with compact support in Darboux coordinates, using formulas (12) and (13) and intergration by parts, we then get

$$
D=-2 a,
$$

where $a: C^{\infty}(M) \rightarrow C^{\infty}(M)$ is the unique $L^{2}$-symmetric differential operator on $C^{\infty}(M)$ with respect to symplectic volume satisfying the coboundary formula (11). In light of Example 3.3, this fact generalizes the KarabegovSchlichenmaier expansion [27, 24] for the Berezin-Toeplitz quantizations of Theorem 2.7 .

Another consequence of the improvement (28) of the reversibility property (P5) is that "unsharpness equals variance on coherent states". To see this, recall definition (22) of the Husimi measure on the coherent state $F_{\hbar, x} \in$ $\mathcal{S}\left(H_{\hbar}\right)$ of Remark 2.2. Then the discussion above implies

$$
\begin{aligned}
\operatorname{Var}\left(f, \mu_{F_{x, \hbar}}\right) & =\mathcal{B}_{\hbar}\left(f^{2}\right)-\mathcal{B}_{\hbar}(f)^{2} \\
& =-2 \hbar c^{+}(f, f)+\mathcal{O}\left(\hbar^{2}\right) .
\end{aligned}
$$

Thus by formula (23) and (26), we get

$$
\operatorname{Var}\left(T_{\hbar}(f), F_{\hbar, x}\right)=-\hbar c_{+}(f, f)(x)+\mathcal{O}\left(\hbar^{2}\right),
$$

so that the variance of a quantized observable at coherent states is equal to its unsharpness.

In their geometric formulation of quantum mechanics, Ashtekar and Shilling [3. $\S 3.2 .3,(26)$ ] consider the projectivization $\mathbb{P}\left(H_{\hbar}\right)$ as a "quantum phase space": a line $\xi \in \mathbb{P}\left(H_{\hbar}\right)$ is identified with the pure state given by the rankone projector to $\xi$. In this setting, they give a physical interpretation of the Fubini-Study metric $g^{F S}$ on $\mathbb{P}\left(H_{\hbar}\right)$ in terms of the variance of a quantum observable $A \in \mathcal{L}\left(H_{\hbar}\right)$ at a pure state $\xi \in \mathbb{P}\left(H_{\hbar}\right)$. Specifically, write $v_{A}$ for the vector field on $\mathbb{P}\left(H_{\hbar}\right)$ induced by the infinitesimal action of $i A \in \mathfrak{u}\left(H_{\hbar}\right)$, seen as an element of the Lie algebra of the group of unitary operators $U\left(H_{\hbar}\right)$ acting on $\mathbb{P}\left(H_{\hbar}\right)$. Then the variance of $A$ at $\xi$ is given by

$$
\operatorname{Var}(A, \xi)=\frac{1}{2} g_{\xi}^{F S}\left(v_{A}, v_{A}\right) .
$$

Back to the quantization, assume further that the coherent states $F_{\hbar, x} \in$ $\mathcal{S}\left(H_{\hbar}\right)$ are pure for all $x \in M$. Consider the induced map

$$
F_{\hbar}: M \longrightarrow \mathbb{P}\left(H_{\hbar}\right) .
$$


Then equation (33) says that the Fubini-Study length of the vector field $v_{A}$ induced by the quantum observable $A:=T_{\hbar}(f) \in \mathcal{L}\left(H_{\hbar}\right)$ at the coherent state $F_{\hbar, x} \in \mathbb{P}\left(H_{\hbar}\right)$ approaches, as $\hbar \rightarrow 0$, the length of the Hamiltonian vector field sgrad $f$ at a point $x \in M$ with respect to our unsharpness metric. In the case of the Kähler quantizations of Theorem 2.7, the map (35) coincides with the Kodaira map. Then the picture described above is closely related to a theorem of Tian [37] showing that the pullback of the Fubini-Study metric by the Kodaira map approaches the Khler metric as $k \rightarrow+\infty$.

\section{Proof of the main Theorem}

In this Section, we prove Theorem 4.1. To this end, first recall from the previous section that the non-negativity of the noise operator (24) leads to the semi-positivity property (27) for the unsharpness. To establish the stronger statement (i) of Theorem 4.1, we will use a stronger property of noise operators coming from the general theory of POVM-based quantum measurements, called the noise inequality. It appears in several sources [30], [21, Theorem 7.5], [31, Theorem 9.4.16], albeit none of them contains the version we need. For the sake of completeness, we present a proof which closely follows [31] and is based on an idea from [25].

Consider a set $M$ equipped with a $\sigma$-algebra $\mathcal{C}$ together with a finitedimensional Hilbert space $H$, and let $F$ be an $\mathcal{L}(H)$-valued POVM in the sense of Definition 2.1. For a bounded function $u \in L^{\infty}(M)$, we define the noise operator

$$
\Delta_{F}(u):=\int_{M} u^{2} d F-\left(\int_{M} u d F\right)^{2} .
$$

For a pair of bounded functions $u, v \in L^{\infty}(M)$, set

$$
U:=\int_{M} u d F, \quad V:=\int_{M} v d F
$$

Lemma 5.1. For every state $\theta \in \mathcal{S}(H)$, we have the inequality

$$
\operatorname{tr}\left(\Delta_{F}(u) \theta\right) \operatorname{tr}\left(\Delta_{F}(v) \theta\right) \geq \frac{1}{4}|\operatorname{tr}([U, V] \theta)|^{2} .
$$


Proof. By the Naimark dilation theorem (see e.g. [31, Theorem 9.4.6]), there exists an isometric embedding of $H$ into a (possibly infinite-dimensional) Hilbert space $H^{\prime}$ and an $\mathcal{L}\left(H^{\prime}\right)$-valued projector valued measure $P$ such that for every subset $X \subset \mathcal{C}$, we have

$$
F(X)=\Pi P(X) \Pi^{*} \in \mathcal{L}(H),
$$

where $\Pi: H^{\prime} \rightarrow H$ is the orthogonal projector, so that $\Pi^{*}: H \rightarrow H^{\prime}$ is the inclusion. Here $\mathcal{L}\left(H^{\prime}\right)$ stands for the space of all bounded Hermitian operators on $H^{\prime}$, and an $\mathcal{L}\left(H^{\prime}\right)$-projector valued measure is by definition a map $P: \mathcal{C} \rightarrow \mathcal{L}\left(H^{\prime}\right)$ satisfying the axioms of Definition 2.1, and such that the operators $P(X), X \in \mathcal{C}$, are pair-wise commuting orthogonal projectors.

Define a pairing

$$
\begin{gathered}
q: \mathcal{L}\left(H^{\prime}\right) \times \mathcal{L}\left(H^{\prime}\right) \rightarrow \operatorname{End}(H), \\
q(S, T):=\Pi S\left(1-\Pi^{*} \Pi\right) T \Pi^{*} .
\end{gathered}
$$

We claim that for every state $\theta \in \mathcal{S}(H)$ and all $S, T \in \mathcal{L}\left(H^{\prime}\right)$, we have

$$
\operatorname{tr}(q(S, S) \theta) \operatorname{tr}(q(T, T) \theta) \geq|\operatorname{tr}(q(S, T) \theta)|^{2} .
$$

To see this, note that $1-\Pi^{*} \Pi: H^{\prime} \rightarrow H^{\prime}$ is the orthogonal projector on the orthogonal complement of $H$, so that

$$
\operatorname{tr}(q(S, T) \theta)=\operatorname{tr}\left(\left(1-\Pi^{*} \Pi\right) T \Pi^{*} \theta \Pi S\left(1-\Pi^{*} \Pi\right)\right) .
$$

Then (38) follows from Cauchy-Schwarz inequality applied to the semi-norm on the space $\operatorname{End}\left(H^{\prime}\right)$ of bounded operators of $H^{\prime}$ defined by $(A, B)_{\theta}:=$ $\operatorname{tr}\left(A \Pi^{*} \theta \Pi B^{*}\right)$, for all $A, B \in \operatorname{End}\left(H^{\prime}\right)$.

Set now $S=\int u d P$ and $T=\int v d P$. Since $S$ and $T$ commute, we have $\left[\Pi S \Pi^{*}, \Pi T \Pi^{*}\right]=q(T, S)-q(S, T)$. On the other hand, by definition (37), we have $\Pi S \Pi^{*}=U$ and $\Pi T \Pi^{*}=V$, while using an approximation by simple functions, one computes that $q(S, S)=\Delta_{F}(u)$ and $q(T, T)=\Delta_{F}(v)$. The statement of the lemma then directly follows from (38).

Proof of (I): Applying Lemma 5.1 to the Berezin-Toeplitz POVM $\Gamma_{\hbar}$ of Definition 2.6. for every state $\theta \in \mathcal{S}\left(H_{\hbar}\right)$ and observables $u, v \in C^{\infty}(M)$, we get

$$
\operatorname{tr}\left(\Delta_{\hbar}(u) \theta\right) \operatorname{tr}\left(\Delta_{\hbar}(v) \theta\right) \geq \frac{1}{4}\left|\operatorname{tr}\left(\left[T_{\hbar}(u), T_{\hbar}(v)\right] \theta\right)\right|^{2}
$$


Now by Definition 2.4 of the Berezin transform and the expression (24) for the noise operator, we know that for all $u \in C^{\infty}(M)$,

$$
\operatorname{tr}\left(\Delta_{\hbar}(u) F_{\hbar, x}\right)=-\hbar \mathcal{B}\left(c_{+}(u, u)\right)(x)+\mathcal{O}\left(\hbar^{2}\right),
$$

and for all $u, v \in C^{\infty}(M)$,

$$
\begin{aligned}
-i \operatorname{tr}\left(\left[T_{\hbar}(u), T_{\hbar}(v)\right] F_{\hbar, x}\right) & =\hbar \operatorname{tr}\left(T_{\hbar}(\{u, v\}) F_{\hbar, x}\right)+\mathcal{O}\left(\hbar^{2}\right) \\
& =\hbar B_{\hbar}(\{u, v\})(x)+\mathcal{O}\left(\hbar^{2}\right) .
\end{aligned}
$$

Thus applying the noise inequality (39) with $\theta$ being the coherent state $F_{\hbar, x}$, we get that

$$
\mathcal{B}_{\hbar}\left(c_{+}(u, u)\right)(x) \mathcal{B}_{\hbar}\left(c_{+}(v, v)\right)(x) \geq \frac{1}{4}\left|\mathcal{B}_{\hbar}(\{u, v\})(x)\right|^{2},
$$

so that the reversibility property (P5) yields

$$
c_{+}(u, u) c_{+}(v, v) \geq \frac{1}{4}|\{u, v\}|^{2} .
$$

Thus for all $\xi, \eta \in T_{x} M$, picking functions $u, v \in C^{\infty}(M)$ with $\operatorname{sgrad} u(x)=$ $\xi, \operatorname{sgrad} v(x)=\eta$ and by definition (20) of the bilinear form $G$, we get

$$
G(\xi, \xi) G(\eta, \eta) \geq|\omega(\xi, \eta)|^{2} .
$$

Now thanks to the non-negativity of the noise operator, which follows from Lemma 5.1, we already know that $G$ is a semi-positive symmetric bilinear form by formula (27). Inequality (44) then shows that $G$ is in fact positive, so that it defines a Riemannian metric on $M$.

Let $K \in \operatorname{End}(T M)$ the $G$-antisymmetric operator defined by

$$
G(\cdot, \cdot)=\omega(\cdot, K \cdot)
$$

Then there exists an orthonormal basis $\left\{e_{j}, f_{j}\right\}_{1 \leq j \leq \operatorname{dim} M}$ of $T M$ such that $K e_{j}=\alpha_{j} f_{j}$ and $K f_{j}=-\alpha_{j} e_{j}$, for $\alpha_{j} \geq 0$ for all $1 \leq j \leq \operatorname{dim} M$. Define an almost complex structure $J \in \operatorname{End}(T M)$ by the formula

$$
J e_{j}=f_{j} \text { and } J f_{j}=-e_{j} .
$$

By definition, this almost complex structure is compatible with $\omega$, and $G$ is $J$-invariant. Set

$$
\rho(\cdot, \cdot):=G(\cdot, \cdot)-\omega(\cdot, J \cdot)
$$


We then need to show that for any $\xi \in T M$, we have

$$
\rho(\xi, \xi) \geq 0
$$

But using (44), we know that

$$
G(\xi, \xi)=G(\xi, \xi)^{1 / 2} G(J \xi, J \xi)^{1 / 2} \geq \omega(\xi, J \xi),
$$

which readily implies (48) by definition (47) of $\rho$.

Proof OF (II): Recall that the volume of an $\omega$-compatible metric is always equal to the symplectic volume $\operatorname{Vol}(M, \omega)$. Then the statement (II) follows from the general form of an unsharpness metric $G$ given by formula (21).

Proof OF (III): The construction below is a modification of the one in Example 3.4. Instead of dealing with the heat semigroup, which becomes elusive when the form $\rho$ is degenerate, we construct an explicit family of Markov kernels such that the desired quantization is the composition of the almost-Kähler quantization associated with $J$ from formula (21) with the corresponding Markov operator. 2 Let us pass to precise arguments.

All the estimates in the proof are meant uniformly in $x_{0} \in M$. Let $J \in \operatorname{End}(T M)$ be a compatible almost complex structure on $(M, \omega)$ and let $\rho$ be a non-negative symmetric bilinear form on $T M$. Consider the Riemannian metric $g$ over $M$ defined by the formula

$$
g(\cdot, \cdot)=\omega(\cdot, J \cdot)
$$

For any $t>0$, we define a smooth endomorphism of the tangent bundle $T M$ by the formula

$$
A_{t}:=t\left(-\pi J \rho_{g} J+t 1\right) \in \operatorname{End}(T M),
$$

where $\rho_{g} \in \operatorname{End}(T M)$ is the non-negative symmetric endomorphism defined by

$$
g\left(\rho_{g} \cdot, \cdot\right)=\rho .
$$

Then $A_{t}$ is positive symmetric with respect to $g$, for all $t>0$.

Let $\epsilon>0$ be smaller than the injectivity radius of $(X, g)$. For any $x_{0} \in X$, consider an isometric identification $\left(T_{x_{0}} X, g\right) \simeq\left(\mathbb{R}^{2 d},\langle\cdot, \cdot\rangle\right)$, where $\langle\cdot, \cdot\rangle$ is

\footnotetext{
${ }^{2}$ In the language of quantum measurement theory, the POVM of the quantization constructed below is a smearing of the Berezin-Toeplitz POVM of Theorem 2.7 by the explicitly constructed Markov operator.
} 
the standard Euclidean product of $\mathbb{R}^{2 d}$, and let $Z=\left(Z_{1}, \cdots Z_{2 d}\right) \in \mathbb{R}^{2 d}$ be the induced normal coordinates on the geodesic ball $B\left(x_{0}, \epsilon\right) \subset X$ of radius $\epsilon$ centered at $x_{0}$. We write $d Z$ for the Lebesgue measure on $\mathbb{R}^{2 d}$. Let $\varphi:[0,+\infty) \rightarrow[0,1]$ be a smooth function identically equal to 1 over $[0, \epsilon / 2)$ and to 0 over $[\epsilon,+\infty)$. We define an operator $K_{t}^{\rho}$ acting on $f \in C^{\infty}(X, \mathbb{R})$ by the following formula in normal coordinates around $x_{0} \in X$,

$$
K_{t}^{\rho} f\left(x_{0}\right):=\frac{1}{\alpha_{t}\left(x_{0}\right)} \int_{B\left(x_{0}, \epsilon\right)} \varphi(|Z|) f(Z) e^{-\pi\left\langle A_{t}^{-1} Z, Z\right\rangle} d Z,
$$

where $\alpha_{t}\left(x_{0}\right):=\int_{B\left(x_{0}, \epsilon\right)} \varphi(|Z|) e^{-\pi\left\langle A_{t}^{-1} Z, Z\right\rangle} d Z$ is chosen so that $K_{t} 1 \equiv 1$ for all $t>0$. Note that $f \geq 0$ implies $K_{t} f \geq 0$ for all $t>0$.

Fix $x_{0} \in X$, and consider the isometric identification $\left(T_{x_{0}} X, g\right) \simeq\left(\mathbb{R}^{2 d},\langle\cdot, \cdot\rangle\right)$ in which $A_{t}$ is diagonal, so that using definition (51), we can write

$$
A_{t, x_{0}}=\operatorname{diag}\left(t\left(\lambda_{1}+t\right), \cdots, t\left(\lambda_{2 d}+t\right)\right),
$$

where $\left\{\lambda_{j} \geq 0\right\}_{1 \leq j \leq 2 d}$ are the eigenvalues of $-\pi J \rho_{g} J$ over $T_{x_{0}} X$. Using the multi-index notation $\alpha=\left(\alpha_{1}, \cdots, \alpha_{2 d}\right) \in \mathbb{N}^{2 d}$, we will use the following Taylor expansion of $f$ up to order 4 as $|Z| \rightarrow 0$,

$$
f(Z)=\sum_{0 \leq|\alpha| \leq 3} \frac{\partial^{|\alpha|} f}{\partial Z^{\alpha}}\left(x_{0}\right) \frac{Z^{\alpha}}{\alpha !}+O\left(|Z|^{4}\right)\|f\|_{C^{4}} .
$$

On the other hand, using the change of variables $Z_{j} \mapsto Z_{j} / t^{1 / 2}\left(\lambda_{j}+t\right)^{1 / 2}$ for each $1 \leq j \leq 2 d$ and the exponential decrease of the Gaussian function, we get a constant $\delta>0$ for any $\alpha \in \mathbb{N}^{2 d}$ such that the following estimate holds as $t \rightarrow 0$,

$$
\begin{gathered}
\int_{B\left(x_{0}, \epsilon\right)} \varphi(|Z|) Z^{\alpha} e^{-\pi\left\langle A_{t}^{-1} Z, Z\right\rangle} d Z=\int_{\mathbb{R}^{2 d}} Z^{\alpha} e^{-\pi \sum_{j=1}^{2 d}\left(t^{-1}\left(\lambda_{j}+t\right)^{-1} Z_{j}^{2}\right)} d Z \\
-\int_{\mathbb{R}^{2 d}}(1-\varphi(|Z|)) Z^{\alpha} e^{-\pi \sum_{j=1}^{2 d}\left(t^{-1}\left(\lambda_{j}+t\right)^{-1} Z_{j}^{2}\right)} d Z \\
=\prod_{j=1}^{2 d} t^{1 / 2}\left(\lambda_{j}+t\right)^{1 / 2}\left(t\left(\lambda_{j}+t\right)\right)^{\alpha_{j} / 2} \int_{\mathbb{R}^{2 d}} Z^{\alpha} e^{-\pi|Z|^{2}} d Z+O\left(e^{-\delta / t}\right) .
\end{gathered}
$$

Note that we can then explicitly evaluate the integral in the last line of (56) using basic properties of the Gaussian function, and it vanishes as soon as 
there is an odd monomial inside $Z^{\alpha}$. Then considering the Taylor expansion (55) inside the right hand side of equation (53) and using the estimate (56), we get as $t \rightarrow 0$,

$$
\begin{aligned}
K_{t}^{\rho} f & \left(x_{0}\right)=f\left(x_{0}\right) \\
& +\frac{\prod_{k=1}^{2 d} t^{1 / 2}\left(\lambda_{k}+t\right)^{1 / 2}}{\alpha_{t}\left(x_{0}\right)}\left(\sum_{j=1}^{2 d} \frac{t\left(\lambda_{j}+t\right)}{4 \pi} \frac{\partial^{2} f}{\partial Z_{j}^{2}}\left(x_{0}\right)+O\left(t^{2}\right)\|f\|_{C^{4}}\right) .
\end{aligned}
$$

On the other hand, it follows from the definition of $\alpha_{t}$ and the estimate (56) that as $t \rightarrow 0$, we have

$$
\alpha_{t}\left(x_{0}\right)=\prod_{j=1}^{2 d} t^{1 / 2}\left(\lambda_{j}+t\right)^{1 / 2}\left(1+O\left(e^{-\delta / t}\right)\right) .
$$

Then we get from equation (157) that as $t \rightarrow 0$,

$$
\begin{aligned}
K_{t}^{\rho} f\left(x_{0}\right) & =f\left(x_{0}\right)+t \sum_{j=1}^{2 d} \frac{\lambda_{j}+t}{4 \pi} \frac{\partial^{2} f}{\partial Z_{j}^{2}}\left(x_{0}\right)+O\left(t^{2}\right)\|f\|_{C^{4}} \\
& =f\left(x_{0}\right)+t \sum_{j=1}^{2 d} \frac{\lambda_{j}}{4 \pi} \frac{\partial^{2} f}{\partial Z_{j}^{2}}\left(x_{0}\right)+O\left(t^{2}\right)\|f\|_{C^{4}} .
\end{aligned}
$$

Then writing $T_{\hbar}$ for the Berezin-Toeplitz quantization of $(M, \omega, J)$, the quantization $T_{\hbar}^{\rho}$ defined for all $f \in C^{\infty}(X, \mathbb{R})$ by

$$
T_{\hbar}^{\rho}(f):=T_{\hbar}\left(K_{\hbar}^{\rho} f\right),
$$

has unsharpness metric $G$ given by formula (21): in fact, for any $u, v \in$ $C^{\infty}(X, \mathbb{R})$, writing $\nabla_{g} u, \nabla_{g} v$ for their gradient with respect to $g$ and in normal coordinates around $x_{0} \in X$ as above, we get from the last line of (59) and following the computations of (18) that the unsharpness cocycle $c_{+}^{\rho}$ associated with $T_{\hbar}^{\rho}$ satisfies

$$
\begin{aligned}
c_{+}^{\rho}(u, v)\left(x_{0}\right) & =-\frac{1}{2} \sum_{j=1}^{2 d}\left(\partial_{j} u\left(x_{0}\right) \partial_{j} v\left(x_{0}\right)+\frac{\lambda_{j}}{\pi} \partial_{j} u\left(x_{0}\right) \partial_{j} v\left(x_{0}\right)\right) \\
& =-\frac{1}{2}\left(g_{x_{0}}\left(\nabla_{g} u, \nabla_{g} v\right)-g_{x_{0}}\left(J \rho_{g} J \nabla_{g} u, \nabla_{g} v\right)\right) \\
& =-\frac{1}{2}\left(g_{x_{0}}(\operatorname{sgrad} u, \operatorname{sgrad} v)+\rho_{x_{0}}(\operatorname{sgrad} u, \operatorname{sgrad} v)\right) .
\end{aligned}
$$

This shows that $G=g+\rho$, as required. 


\section{Case study: $S U(2)$ - equivariant quantiza- tions}

Definition 6.1. Two Berezin-Toeplitz quantizations $T_{\hbar}$ and $T_{\hbar}^{\prime}$ with families of Hilbert spaces $\left\{H_{\hbar}\right\}$ and $\left\{H_{\hbar}^{\prime}\right\}, \hbar \in \Lambda$, respectively, are called equivalent if there exists a sequence of unitary operators $U_{\hbar}: H_{\hbar} \rightarrow H_{\hbar}^{\prime}$ such that for all $f \in C^{\infty}(M)$,

$$
\left\|U_{\hbar} T_{\hbar}(f) U_{\hbar}^{-1}-T_{\hbar}^{\prime}(f)\right\|_{o p}=\mathcal{O}\left(\hbar^{2}\right) .
$$

Observe that if two quantizations are equivalent, their unsharpness metrics coincide. In this section we prove a converse statement in the context of $S U(2)$-equivariant quantizations of the two-dimensional sphere (see Section 8.3 below for further discussion). We consider the standard Kähler metric on the two-sphere $S^{2}$ normalized so that the total area equals $2 \pi$. We denote by $L$ the line bundle dual to the tautological one, and by $H_{k}$ the $k+1$ dimensional space of holomorphic sections of its $k$-th tensor power $L^{k}$. One can identify $H_{k}$ with the space of homogeneous polynomials of two variables, so the group $S U(2)$ acts on $H_{k}$ via an irreducible unitary representation. Furthermore, $S U(2)$ acts on the space of Hermitian operators $\mathcal{L}\left(H_{k}\right)$ by conjugation. On the other hand the space $C^{\infty}\left(S^{2}\right)$ carries the natural action of $S U(2)$ by the change of variables. A quantization $Q_{\hbar}: C^{\infty}\left(S^{2}\right) \rightarrow \mathcal{L}\left(H_{k}\right)$, $\hbar \in \Lambda:=\{1 / k\}_{k \in \mathbb{N}}$, is called $S U(2)$-equivariant if it intertwines the corresponding (real) representations. For instance, the standard Berezin-Toeplitz quantization $T_{\hbar}$ sending $f \in C^{\infty}\left(S^{2}\right)$ to the multiplication by $f$ followed by the orthogonal projection to the space of holomorphic sections is $S U(2)$ equivariant, and the same holds true for its images $T_{\hbar}^{(t)}$ under diffusion as defined in Example 3.4. Note that the quantizations $T_{\hbar}^{(t)}$ are pair-wise nonequivalent for different values of $t$ as the corresponding unsharpness metrics are different.

Theorem 6.2. Every $S U(2)$-equivariant quantization of $S^{2}$ is equivalent to $T_{\hbar}^{(t)}$ for some $t \geq 0$.

Proof. Step 1 (Applying Schur lemma): Given any $S U(2)$-equivariant quantization $Q_{\hbar}$, pass to its complexification (denoted by the same letter)

$$
Q_{\hbar}: C^{\infty}\left(S^{2}, \mathbb{C}\right) \rightarrow \mathcal{L}\left(H_{k}\right) \otimes \mathbb{C}=H_{k}^{*} \otimes H_{k}
$$


On the one hand, $C^{\infty}\left(S^{2}, \mathbb{C}\right)$ splits into the direct sum of irreducible summands $V_{j}, j=0,1, \ldots$ corresponding to the eigenspaces of the LaplaceBeltrami operator associated to the Kähler metric with the eigenvalue $2 j(j+$ 1 ), with each $V_{j}$ isomorphic to $H_{2 j}$ as an $S U(2)$-representation. On the other hand

$$
H_{k}^{*} \otimes H_{k}=H_{2 k} \oplus H_{2 k-2} \oplus \cdots \oplus H_{0} .
$$

By the Schur Lemma, when $\hbar=1 / k$, we have that $Q_{\hbar}\left(V_{j}\right) \subset H_{2 j}$ with respect to this decomposition, and furthermore there exists a constant $\alpha_{\hbar, j} \in \mathbb{C}$ such that, up to conjugation, we have

$$
Q_{\hbar}=\left(1+\alpha_{\hbar, j}\right) T_{\hbar} \text { on } V_{j}
$$

STEP 2 (Legendre Polynomials): In what follows we introduce another parameter, $n \in \mathbb{N}$. We call a sequence $\left\{b_{\hbar, n}\right\}_{n \in \mathbb{N}}$ of the class $\mathcal{O}_{N}\left(\hbar^{m}\right)$ with $m, N \in \mathbb{N}$ if for some $c>0$ we have $\left|b_{\hbar, n}\right| \leq c \hbar^{m}(n+1)^{N}$ for all $n$. In the sequel, the dependence on $\hbar$ of such sequences will be made implicit. Denote by $P_{n}(z)$ the $n$-th Legendre polynomial considered as a function on the unit sphere $S^{2}=\left\{x^{2}+y^{2}+z^{2}=1\right\}$ lying in $V_{n}$. We write $\nabla$ for the gradient with respect to the standard metric on $S^{2}$ normalized so that the total area equals $2 \pi$. The standard formulas for the Legendre polynomials (see e.g. formulas (43) and (44) in [38]) readily yield,

$$
P_{1} P_{n}=q_{n} P_{n+1}+r_{n} P_{n-1}, \quad q_{n}=\frac{n+1}{2 n+1}, \quad r_{n}=1-q_{n},
$$

and

$$
\left(\nabla P_{1}, \nabla P_{n}\right)=s_{n}\left(-P_{n+1}+P_{n-1}\right), s_{n}=\frac{2 n(n+1)}{2 n+1} .
$$

We shall use that there exists $c>0$ such that

$$
\forall r \in \mathbb{N} \exists R \in \mathbb{N}:\left\|P_{n}\right\|_{C^{r}} \leq c(n+1)^{R}
$$

This (with $R=r$ ) follows immediately from the general result about the growth of $C^{r}$-norms of the Laplace-Beltrami eigenfunctions on Riemannian manifolds, see [8, Corollary 1.1]. Using the fact that $\max _{x \in S^{2}} P_{n}=1$ by [26, Chapter 7, Theorem 17(i)], the norm correspondence property (P1), which holds uniformly in $C^{N}$-norm for some $N \in \mathbb{N}$, together with formula (66) implies

$$
\left\|Q_{\hbar}\left(P_{n}\right)\right\|_{o p}=1-\mathcal{O}_{N}(\hbar),\left\|T_{\hbar}\left(P_{n}\right)\right\|_{o p}=1-\mathcal{O}_{N}(\hbar)
$$


Since $Q_{\hbar}\left(P_{n}\right)=\left(1+\alpha_{\hbar, n}\right) T_{\hbar}\left(P_{n}\right)$ by (63)), it follows that

$$
\alpha_{\hbar, n}=\mathcal{O}_{N}(\hbar) .
$$

In the course of the proof, we shall increase the value of $N$ according to our needs.

Step 3 (Main Calculation) : Since $Q_{\hbar}$ is $S U(2)$-equivariant, the corresponding unsharpness metric equals $\mu$ times the standard one, for some constant $\mu \geq 1$. Thus the quasi-multiplicativity property (P3), which holds uniformly in $C^{N}$-norm for some $N \in \mathbb{N}$, together with formula (66) yields

$$
Q_{\hbar}\left(P_{1}\right) Q_{\hbar}\left(P_{n}\right)=Q_{\hbar}\left(P_{1} P_{n}-\frac{\mu}{2} \hbar\left(\nabla P_{1}, \nabla P_{n}\right)+\mathcal{O}_{N}\left(\hbar^{2}\right)\right) .
$$

At the same time

$$
T_{\hbar}\left(P_{1}\right) T_{\hbar}\left(P_{n}\right)=T_{\hbar}\left(P_{1} P_{n}-\frac{1}{2} \hbar\left(\nabla P_{1}, \nabla P_{n}\right)+\mathcal{O}_{N}\left(\hbar^{2}\right)\right),
$$

mind that here $\mu$ is replaced by 1 . By (63) we have

$$
Q_{\hbar}\left(P_{i}\right)=\left(1+\alpha_{\hbar, i}\right) T_{\hbar}\left(P_{i}\right) .
$$

Identities (68) and (69) combined with (64), (65) and (70) enable us to express $T_{\hbar}\left(P_{1}\right) T_{\hbar}\left(P_{n}\right)$ as a linear combination of $T_{\hbar}\left(P_{n+1}\right)$ and $T_{\hbar}\left(P_{n-1}\right)$ in two different ways. The calculation is straightforward, and we obtain the result:

$$
A_{n} T_{\hbar}\left(P_{n+1}\right)+B_{n} T_{\hbar}\left(P_{n-1}\right)=A_{n}^{\prime} T_{\hbar}\left(P_{n+1}\right)+B_{n}^{\prime} T_{\hbar}\left(P_{n-1}\right)+\mathcal{O}_{N}\left(\hbar^{2}\right),
$$

for some $A_{n}, A_{n}^{\prime} \in \mathbb{C}$ and $B_{n}, B_{n}^{\prime} \in \mathbb{C}, n \in \mathbb{N}$, where

$$
\begin{gathered}
B_{n}=\left(1+\alpha_{\hbar, 1}\right)^{-1}\left(1+\alpha_{\hbar, n}\right)^{-1}\left(1+\alpha_{\hbar, n-1}\right)\left(r_{n}-\hbar \mu s_{n} / 2\right), \\
B_{n}^{\prime}=r_{n}-\hbar s_{n} / 2 .
\end{gathered}
$$

Projecting equation (71) to the space $H_{2 n-2}$ (which contains $T_{\hbar}\left(V_{n-1}\right)$ ) and using that the operator norm of $T_{\hbar}\left(P_{n-1}\right)$ is bounded away from zero (see Step 2), we get that

$$
B_{n}-B_{n}^{\prime}=\mathcal{O}_{N}\left(\hbar^{2}\right) .
$$

By using (67) and explicit expressions for $q_{n}, r_{n}, s_{n}$ we get

$$
\alpha_{\hbar, n-1}-\alpha_{\hbar, n}-\alpha_{\hbar, 1}=(n+1)(\mu-1) \hbar+\mathcal{O}_{N}\left(\hbar^{2}\right) .
$$


Substituting $n=1$ into (72) we get that

$$
\alpha_{\hbar, 1}=-(\mu-1) \hbar+\mathcal{O}_{N}\left(\hbar^{2}\right) .
$$

Now we get a recursive formula

$$
\alpha_{\hbar, n}=\alpha_{\hbar, n-1}-n(\mu-1) \hbar+\mathcal{O}_{N}\left(\hbar^{2}\right) .
$$

Noticing that $(n+1) \mathcal{O}_{N}\left(\hbar^{2}\right)=\mathcal{O}_{N+1}\left(\hbar^{2}\right)$ and redefining $N \mapsto N+1$ we conclude that

$$
\alpha_{\hbar, n}=-\frac{n(n+1)}{2}(\mu-1) \hbar+\mathcal{O}_{N}\left(\hbar^{2}\right) .
$$

SteP 4 (FInAle) : Recall that $2 n(n+1)$ is the eigenvalue of the Laplacian corresponding to the eigenspace $V_{n}$. Let $V=\oplus_{n=0}^{\infty} V_{n}$ be the space of all finite linear combinations of spherical harmonics. By norm correspondence (P1) and formula (73), for every $\phi_{n} \in V_{n}$ we have

$$
\begin{aligned}
& Q_{\hbar}\left(\phi_{n}\right)=\left(1-\frac{n(n+1)}{2}(\mu-1) \hbar\right) T_{\hbar}\left(\phi_{n}\right)+\mathcal{O}_{N}\left(\hbar^{2}\right)\left\|\phi_{n}\right\|_{C^{N}} \\
& =T_{\hbar}\left(e^{-t \hbar \Delta} \phi_{n}\right)+\mathcal{O}_{N}\left(\hbar^{2}\right)\left\|\phi_{n}\right\|_{C^{N}}=T_{\hbar}^{(t)}\left(\phi_{n}\right)+\mathcal{O}_{N}\left(\hbar^{2}\right)\left\|\phi_{n}\right\|_{C^{N}}
\end{aligned}
$$

with $t=(\mu-1) / 4$ in Example 3.4 .

Take now any $f \in C^{\infty}\left(S^{2}\right)$, and decompose it by spherical harmonics: $f=\sum_{n} \phi_{n}$. Since $f$ is smooth, the $C^{N}$-norms $\left\|\phi_{n}\right\|_{C^{N}}$ decay faster than any power of $n$ as $n \rightarrow+\infty$, so that

$$
\left\|Q_{\hbar}(f)-T_{\hbar}^{(t)}(f)\right\|_{o p} \leq c \sum_{n \in \mathbb{N}} n^{N}\left\|\phi_{n}\right\|_{C^{N}} \hbar^{2} \leq c^{\prime} \hbar^{2} .
$$

This shows that the quantizations $Q_{\hbar}$ and $T_{\hbar}^{(t)}$ are equivalent.

\section{The unsharpness cocycle is of order $(1,1)$}

In this Section we prove Theorem 3.2

Proof. For every $d \in \mathbb{N}$, we use the standard multi-index notation $\alpha=$ $\left(\alpha_{1}, \cdots, \alpha_{d}\right) \in \mathbb{N}^{d}$, where for any sequence of symbols $x_{1}, \cdots, x_{d}$, we write $x_{\alpha}:=x_{\alpha_{1}} \cdots x_{\alpha_{d}}$, so that in particular $\alpha !:=\alpha_{1} ! \cdots \alpha_{d} ! \in \mathbb{N}$, and write $|\alpha|:=\alpha_{1}+\cdots+\alpha_{d}$ 
Note that by (11), to show that $c_{+}$is a bi-differential operator of bi-degree $(1,1)$, we need to show that the differential operator a contains only terms of order 1 and 2 . Note that $T_{\hbar}(1)=1$ implies $c_{+}(1,1)=0$, so that $a$ cannot contain terms of order 0 . Let us show that $a$ cannot be of order $k>2$.

Assume by contradiction that $a$ is of order $k>2$. Let $x_{0} \in X$ be the center of local coordinates $\left(Z_{1}, \cdots, Z_{2 n}\right) \in U \subset \mathbb{R}^{2 n}$ be such that for all $f \in C^{\infty}(X, \mathbb{R})$,

$$
a f\left(x_{0}\right)=\sum_{1 \leq|\alpha| \leq k} a_{\alpha} \frac{\partial^{|\alpha|} f}{\partial Z^{\alpha}}\left(x_{0}\right),
$$

where the sequence $\left\{a_{\alpha} \in \mathbb{R}\right\}_{1 \leq|\alpha| \leq k}$ is such that $a_{\beta} \neq 0$ for some $\beta=$ $\left(\beta_{1}, \cdots, \beta_{2 n}\right) \in \mathbb{N}^{2 n}$ of length $|\beta|=k$. Fix $1 \leq j \leq 2 n$ such that $\beta_{j} \neq 0$, and writing $\hat{\beta}=\left(\beta_{1}, \cdots, \beta_{j}-1, \cdots, \beta_{2 n}\right) \in \mathbb{N}^{2 n-1}$, take $f \in C^{\infty}(X, \mathbb{R})$ satisfying

$$
f(Z)=\frac{c}{\hat{\beta} !} Z^{\hat{\beta}}+Z_{j}
$$

for $Z \in U \subset \mathbb{R}^{2 n}$ in the coordinates around $x_{0} \in X$ considered above and for some $c \in \mathbb{R}$ to be fixed later. Then this function $f$ and all its derivatives vanish at $x_{0} \in X$, except for

$$
\frac{\partial^{|\hat{\beta}|} f}{\partial Z^{\hat{\beta}}}\left(x_{0}\right)=c \quad \text { and } \quad \frac{\partial f}{\partial Z_{j}}\left(x_{0}\right)=1 .
$$

Then by equations (75) and (74), considering the multi-index $\gamma \in \mathbb{N}^{2 n}$ of length $|\gamma|=2$ such that $\gamma_{j}=2$, one gets that for any $f \in C^{\infty}(X, \mathbb{R})$ satisfying (76),

$$
\begin{aligned}
c_{+}(f, f)\left(x_{0}\right) & =2 a_{\beta} \frac{\partial^{|\hat{\beta}|} f}{\partial Z^{\hat{\beta}}}\left(x_{0}\right) \frac{\partial f}{\partial Z_{j}}\left(x_{0}\right)+2 a_{\gamma} \frac{\partial f}{\partial Z_{j}}\left(x_{0}\right) \frac{\partial f}{\partial Z_{j}}\left(x_{0}\right) \\
& =2 a_{\beta} c+2 a_{\gamma} .
\end{aligned}
$$

Thus if $f \in C^{\infty}(X, \mathbb{R})$ satisfies (76) for $c \in \mathbb{R}$ such that $\operatorname{sign}\left(a_{\beta}\right) c>-a_{\gamma} /\left|a_{\beta}\right|$, we get that $c_{+}(f, f)\left(x_{0}\right)>0$. This contradicts the fact that $c_{+}(f, f) \leq 0$ for all $f \in C^{\infty}(X, \mathbb{R})$, which holds for every Berezin-Toeplitz quantization by the semi-positivity property (27). 


\section{Discussion and questions}

\subsection{Historical remarks on unsharpness}

The unsharpness cocycle appeared in earlier literature which, to the best of our knowledge, focussed on its elimination, of course, by the price of losing the positivity of a quantization. Let us elaborate on this point. Assume $(M, \omega)$ is a quantizable symplectic manifold equipped with a compatible almost-Kähler structure. Consider the induced Berezin-Toeplitz quantization of Theorem 2.7. Using the notations of Example 3.4, define for any $f \in C^{\infty}(M)$ and $\hbar \in \Lambda=\{1 / k\}_{k \in \mathbb{N}}$,

$$
Q_{\hbar}(f):=T_{\hbar}\left(f+\frac{\hbar}{4} \Delta f\right) .
$$

This gives rise to a collection of maps $Q_{\hbar}: C^{\infty}(M) \rightarrow \mathcal{L}\left(H_{\hbar}\right)$ parametrized by $\hbar \in \Lambda$ and satisfying the axioms (P1)-(P4) of Definition 2.6. but which does not preserve positivity, so that they do not come from a POVM construction via formula (4). Then following the computation (18) in Example 3.3, we see that the associated unsharpness cocycle $c_{+}^{Q}$, defined from the quasimultiplicativity property (P3) as in the beginning of the section, satisfies

$$
c_{+}^{Q}(f, g)=0,
$$

for all $f, g \in C^{\infty}(M)$. As noted for instance by Charles in [13, $\left.\S 1.4\right] 3$, the quantization (78) is, up to twisting with a line bundle, the metaplectic Kostant-Souriau quantization, which possesses remarkable sub-principal properties, a fact which is explained conceptually by the vanishing unsharpness property (79).

In the flat case $M=\mathbb{C}$ with the standard symplectic form, Gerstenhaber considers in [18] deformation quantizations parametrized by $\lambda \geq 0$ which, up to the second order in $\hbar$, correspond to the quantization maps parametrized by $\hbar>0$ defined for any smooth function $f: \mathbb{C} \rightarrow \mathbb{R}$ of polynomial growth by

$$
Q_{\hbar}^{(\lambda)}(f):=T_{\hbar}\left(f+\frac{1-\lambda}{2} \hbar \Delta f\right) .
$$

Here $T_{\hbar}$ is the standard Toeplitz quantization of $\mathbb{C}$, sending $f$ to the multiplication by $f$ followed by the orthogonal $L_{2}$-projection on the space of holo-

\footnotetext{
${ }^{3}$ [13] uses the holomorphic Laplacian, which is half the Laplace-Beltrami operator.
} 
morphic functions which are square integrable with respect to a Gaussian measure. Gerstenhaber formulates a least uncertainty principle for deformation quantization, which implies in particular that unsharpness vanishes on the classical harmonic oscillator. He then shows that the quantization (80) satisfies this least uncertainty principle if $\lambda=1 / 2$, which corresponds to the flat version of the quantization (78).

Note that in the flat case $M=\mathbb{C}$, the classical harmonic oscillator is a sum of squares of the coordinate functions. On the other hand, the quasimultiplicativity property (P3) implies that for all $f \in C^{\infty}(M)$ as $\hbar \rightarrow 0$,

$$
T_{\hbar}(f)^{2}-T_{\hbar}\left(f^{2}\right)=\hbar T_{\hbar}\left(c_{+}(f, f)\right)+\mathcal{O}\left(\hbar^{2}\right) .
$$

We then see that unsharpness measures in particular the deviation of the quantum harmonic oscillator, defined as a sum of squares of the quantum coordinate operators, from the quantization of the classical harmonic oscillator. This explains in particular the standard justification of the metaplectic correction, as giving the "correct" quantum harmonic oscillator on flat space.

\subsection{Least unsharpness surfaces and pseudo-holomorphic curves}

Let $G$ be the unsharpness metric associated to a Berezin-Toeplitz quantization of a closed symplectic manifold $(M, \omega)$ (see Section 4). A least unsharpness surface $\Sigma \subset M$ is a two-dimensional oriented submanifold with $\operatorname{Area}_{G}(\Sigma)=\int_{\Sigma} \omega$. Repeating the the proof of Theorem 4.1 we see that for such surfaces, the restriction of the Riemannian area form coincides with the restriction of the symplectic form. If $G$ has the minimal possible total unsharpness and hence by Theorem 4.1(II) comes from some compatible almost-complex structure $J$ on $M$, the least unsharpness surfaces in $M$ are $J$-holomorphic curves (cf. [33]). For instance, for the complex projective plane $M=\mathbb{C} P^{2}$, Gromov's theory of pseudo-holomorphic curves predicts that for every compatible $J$, through every two distinct points $A, B \in M$ passes unique such curve $\Sigma$ in the homology class of $\left[\mathbb{C} P^{1}\right]$.

It is enticing to interpret $\Sigma$ as a worldsheet of the topological string theory describing a path joining constant loops $A$ and $B$. Note that the metric $G$ on our "space-time" $M$ is canonically associated to a Berezin-Toeplitz quantization of $M$, and the "total unsharpness" $\operatorname{Area}_{G}(\Sigma)$ of a worldsheet $\Sigma$ is nothing else but the Nambu-Goto action up to a multiplicative constant. 
If the total unsharpness of $(M, G)$ is minimal possible, i.e., coincides with the symplectic volume of $M$, the least unsharpness surfaces are $J$-holomorphic curves for a compatible almost complex structure $J$ defining $G$, and hence represent "worldsheet instantons". Does there exist an interpretation of this picture in physical terms?

\subsection{On classification of Berezin-Toeplitz quantizations}

We conclude the paper with a discussion on classification of BerezinToeplitz quantizations up to equivalence in the sense of Definition 6.1. In Section 6 we classified $S U(2)$-equivariant quantizations of the two-dimensional sphere. It would be interesting to extend this to equivariant quantizations for more general co-adjoint orbits equipped with the canonical symplectic structure. In the general (not necessarily equivariant) case, the problem is widely open.

In fact, establishing (non)-equivalence of quantizations is a non-trivial problem even for the Kähler quantizations of Theorem 2.7, where the holomorphic line bundles defining the quantization of $(M, \omega)$ could be non-isomorphic. For instance, their Chern classes could differ by torsion even though the associated spaces of holomorphic sections have same dimension. Are the corresponding quantizations equivalent?

Another interesting example is as follows. According to Remark 4.2, there exist metrics $G$ on $M$ admitting different decompositions of the form (21). Each such decomposition determines a Berezin-Toeplitz quantization given by almost-Kähler quantization followed by diffusion, as explained in the proof of Theorem 4.1(III). Are the quantizations corresponding to different decompositions of the same metric equivalent?

Let us address the question about invariants of quantizations with respect to equivalence. In addition to the unsharpness metric, there is another invariant coming from the trace correspondence, see item (P4) in Definition 2.6. Recall that the latter states that

$$
\operatorname{tr}\left(T_{\hbar}(f)\right)=(2 \pi \hbar)^{-d} \int_{M} f R_{\hbar} d \mu
$$

where $\operatorname{dim} M=2 d$, the function $R_{\hbar} \in C^{\infty}(M)$ satisfies $R_{\hbar}=1+\mathcal{O}(\hbar)$, and $d \mu=\frac{\omega^{d}}{d !}$ is the symplectic volume on $M$. Roughly speaking, since the trace is invariant under conjugation, the convergence rate of the sequence of differential forms $R_{\hbar} d \mu$ to the symplectic volume $d \mu$ as $\hbar \rightarrow 0$ does not 
change up to $\mathcal{O}\left(\hbar^{2}\right)$ under equivalence. For the sake of simplicity, let us, until the end of the paper, enhance axiom (P4) by assuming that there exists a function $r \in C^{\infty}(M)$ such that

$$
R_{\hbar}=1+\hbar r+\mathcal{O}\left(\hbar^{2}\right)
$$

We shall refer to the form $r d \mu$ as the Rawnsley form. Thus, equivalent quantizations possess the same Rawnsley form. Put

$$
\langle r\rangle:=\operatorname{Vol}(M)^{-1} \int_{M} r d \mu
$$

Remark 8.1. Substituting $f=1$ into (P4), we get that $\langle r\rangle$ appears in the dimension formula

$$
\operatorname{dim} H_{\hbar}=\operatorname{Vol}(M)(2 \pi \hbar)^{-d}\left(1+\hbar\langle r\rangle+\mathcal{O}\left(\hbar^{2}\right)\right) .
$$

Let us mention that for Kähler quantizations, an alternative asymptotic expression for the dimension of $H_{\hbar}$ is given by the Hirzebruch-Riemann-Roch theorem. Comparing coefficients at $\hbar$ one gets a simple topological interpretation of $\langle r\rangle$ :

$$
\langle r\rangle=2 \pi \frac{\left\langle[\omega]^{d-1} \cup c_{1}(T M),[M]\right\rangle}{2(d-1) ! \operatorname{Vol}(M)},
$$

where $c_{1}(T M)$ stands for the first Chern class of $M$.

Example 8.2. Let $v$ be a vector field on the manifold $M$ generating a flow $\phi_{t}$. Given a Berezin-Toeplitz quantization $T_{\hbar}$ on $M$, define a new quantization by setting $T_{\hbar}^{(v)}(f):=T_{\hbar}\left(f \circ \phi_{-\hbar}\right)$. A direct calculation based on the expansion $T_{\hbar}^{(v)}(f)=T_{\hbar}(f-\hbar d f(v))+\mathcal{O}\left(\hbar^{2}\right)$ shows that this is a BerezinToeplitz quantization whose unsharpness metric coincides with the one of $T_{\hbar}$, and whose Rawnsley form is given by $(r+\operatorname{div}(v)) d \mu$, where $\operatorname{div}(v)$ stands for the divergence of $v$ with respect to the symplectic volume. In particular, it follows that by choosing an appropriate vector field $v$, one can always achieve the Rawnsley form being equal to $\langle r\rangle$.

Question 8.3. Consider a pair of quantizations with the Hilbert spaces of the same dimension. Suppose that their unsharpness metrics and the Rawnsley forms coincide. Are these quantizations equivalent? 
The answer in the general (not necessarily equivariant) case is at the moment unclear.

Acknowledgement. We thank Pavel Etingof for useful references. We are grateful to Jordan Payette for pointing out a number of inaccuracies and helpful suggestions on the presentation.

\section{References}

[1] Anandan, J., and Aharonov, Y., Geometry of quantum evolution, Phys. Rev. Letters, 65 (1990), p.1697.

[2] Alekseev, A., and Lachowska, A., Invariant *-products on coadjoint orbits and the Shapovalov pairing, Comment. Math. Helv. 80 (2005), 795-810.

[3] Ashtekar, A., and Schilling, T.A., Geometrical Formulation of Quantum Mechanics,, On Einsteins Path, 23-65, Harvey A., Springer, New York, NY, 1999.

[4] Bayen, F., Flato, M., Fronsdal, C., Lichnerowicz, A., and Sternheimer, D., Quantum mechanics as a deformation of classical mechanics, Lett. Math. Phys. 1 (1977), no. 6, 521-530.

[5] Berezin, F., General concept of quantization, Comm. Math. Phys. 40 (1975), 153-174.

[6] Berline, N., Getzler, E., and Vergne, M., Heat kernels and Dirac operators, Grundlehren Text Editions, Springer-Verlag, Berlin, 2004, Corrected reprint of the 1992 original.

[7] Bertelson, M., Bieliavsky, P., and Gutt, S., Parametrizing equivalence classes of invariant star products, Lett. Math. Phys. 46 (1998), 339-345.

[8] Bin, X., Derivatives of the spectral function and Sobolev norms of eigenfunctions on a closed Riemannian manifold, Ann. Global Anal. Geom. 26 (2004), 231-252.

[9] Bordemann, M., Meinrenken, E., and Schlichenmaier, M., Toeplitz quantization of Kähler manifolds and $g l(N), N \rightarrow \infty$ limits, Comm. Math. Phys. 165 (1994), 281-296. 
[10] Borthwick, D., and Uribe, A., Almost complex structures and geometric quantization, Math. Res. Lett. 3 (1996), 845-861.

[11] Boutet de Monvel, L., and Guillemin, V., The spectral theory of Toeplitz operators, Annals of Mathematics Studies, vol. 99, Princeton University Press, Princeton, NJ; University of Tokyo Press, Tokyo, 1981.

[12] Busch, P., Lahti, P., Pellonpää, J.P., and Ylinen, K., Quantum Measurement, Springer, 2016.

[13] Charles, L., Symbolic calculus for Toeplitz operators with half-form, J. Symplectic Geom. 4 (2006), no. 2, 171-198.

[14] Charles, L., Quantization of compact symplectic manifolds, J. Geom. Anal. 26 (2016), no. 4, 2664-2710.

[15] Charles, L., and Polterovich, L., Sharp correspondence principle and quantum measurements, St. Petersburg Math. J., 29 (2018) 177 - 207.

[16] Chiribella, G., D’Ariano, G.M., and Schlingemann, D., How continuous quantum measurements in finite dimensions are actually discrete, Phys. Rev. Lett. 98 (2007), no. 19, 190403.

[17] Dirac, P.A.M., The Principles of Quantum Mechanics, Oxford University Press.

[18] Gerstenhaber, M., Least uncertainty principle in deformation quantization, J. Math. Phys. 48 (2007), no. 2, 022103, 15 pp.

[19] Gromov, M., Pseudo holomorphic curves in symplectic manifolds, Invent. Math. 82 (1985), 307-347.

[20] Guillemin, V., Star products on compact pre-quantizable symplectic manifolds, Lett. Math. Phys. 35 (1995), 85-89.

[21] Hayashi, M., Quantum Information Theory, Graduate Texts in Physics, Springer. 2017.

[22] Ioos, L., On the composition of Berezin-Toeplitz operators on symplectic manifolds, Math. Z. 290 (2018), no. 1-2, 539-559. 
[23] Ioos, L., Lu, W., Ma, X., and Marinescu, G., Berezin-Toeplitz quantization for eigenstates of the Bochner-Laplacian on symplectic manifolds, J. Geom. Anal (2018), 32 pp, DOI 10.1007/s12220-017-9977-y.

[24] Ioos, L., Kaminker, V., Polterovich, L., and Shmoish, D., Spectral aspects of the Berezin transform, arXiv:1811.03155, 2018, to appear in Ann. H. Lebesgue.

[25] Janssens, B., Unifying decoherence and the Heisenberg principle, Lett. Math. Phys. 107 (2017), 1557-1579.

[26] Kaplan, W., Advanced Calculus, 5th edition.

[27] Karabegov, A.V., and Schlichenmaier, M., Identification of BerezinToeplitz deformation quantization, J. Reine Angew. Math. 540 (2001), 49-76.

[28] Klauder, J.R., Quantization is geometry, after all, Ann. Physics 188 (1988), 120-141.

[29] Ma, X., and Marinescu, G., Toeplitz operators on symplectic manifolds, J. Geom. Anal. 18 (2008), 565-611.

[30] Ozawa, M., Quantum limits of measurements and uncertainty principle, in "Quantum Aspects of Optical Communications", 1991, pp. 1-17. Springer, Berlin, Heidelberg.

[31] Polterovich, L., and Rosen, D., Function theory on symplectic manifolds, CRM Monograph Series, 34. American Mathematical Society, Providence, RI, 2014.

[32] Rawnsley, J., and Gutt, S. Equivalence of star products on a symplectic manifold: An introduction to Delignes Cech cohomology classes, J. Geom. Phys. 29, no. 4 (1999), 347-392.

[33] Sanborn, B.A. The uncertainty principle and the energy identity for holomorphic maps in geometric quantum mechanics, arXiv:1710.09344 (2017).

[34] Schlichenmaier, M., Deformation quantization of compact Kähler manifolds by Berezin-Toeplitz quantization, Conférence Moshé Flato 1999, Vol. 
II (Dijon), Math. Phys. Stud., vol. 22, Kluwer Acad. Publ., Dordrecht, 2000, pp. 289-306.

[35] Schlichenmaier, M., Berezin-Toeplitz quantization for compact Kähler manifolds. A review of results, Adv. Math. Phys. (2010), 927280.

[36] Shiffman, B., and Zelditch, S., Asymptotics of almost holomorphic sections of ample line bundles on symplectic manifolds, J. Reine Angew. Math. 544 (2002), 181-222.

[37] Tian, G., On a set of polarized Kähler metrics on algebraic manifolds, J. Differential Geom., 32 (1990), 99-130.

[38] Weisstein, E.W., Legendre Polynomial, From MathWorld-A Wolfram Web Resource. http://mathworld.wolfram.com/LegendrePolynomial.html

[39] Xu, H., An explicit formula for the Berezin star product, Lett. Math. Phys. 101 (2012), 239 - 264.

School of Mathematical Sciences, Tel Aviv University, Ramat Aviv, Tel Aviv 69978 IsRAeL

E-mail address: louisioos@mail.tau.ac.il

Einstein Institute of Mathematics, Hebrew University, Givat Ram, Jerusalem, 91904, ISRAEL

E-mail address: kazhdan@math.huji.ac.il

School of Mathematical Sciences, Tel Aviv University, Ramat Aviv, Tel AVIV 69978 ISRAEL

E-mail address: polterov@tauex.tau.ac.il 